EPJ Web of Conferences 59, 19005 (2013)

DOI: $10.1051 /$ epjconf/20135919005

(C) Owned by the authors, published by EDP Sciences, 2013

\title{
Computational study of microwave-induced ionization process using PIC-MCC method
}

\author{
Yasuyuki Okuno and Naofumi Ohnishi ${ }^{\mathrm{a}}$
}

Department of Aerospace Engineering, Tohoku Univesity, Sendai 980-8579, Japan

\begin{abstract}
We have developed a two-dimensional PIC-MCC code which can describe interactions between input microwave and charged particles and collisions among the gas particles including neutrals in order to examine the mechanism of formation of a filamentary structure observed in a microwave-beaming thruster. Assuming that an initial discharge spot is created by an air-breakdown, an incident microwave is reflected and scattered at the spot, and a strong electric field is formed ahead of it. The region of the strong field is discretisely located at a quarter of microwave wavelength from the original spot as predicted by a simple model, and a newly discharged spot is created through electron impact ionization originating in background electrons in the strong field region.
\end{abstract}

\section{INTRODUCTION}

Although many artificial satellites are launched for space development and business every year, their costs are still huge and prevent space utilization from spreading. Recently, a microwave beaming propulsion was proposed as a low cost launch system. The microwave rocket gains propulsive energy with no fuel by irradiation of high power coherent microwave transmitted from outside of a thruster. The incident microwave is focused inside the thruster and causes an air-breakdown at atmospheric pressure. An ionization front of the induced plasma absorbs the microwave and expands outward at a supersonic speed as sustaining a shock wave. These processes have been investigated experimentally by some authors so far, and they found that the ionization front has filamentary structures [1]. On the other hand, the measured thrust drastically drops down at lower pressures [2]. Any relationship hence may exist between the formation of the filamentary structures and the thrust. Additionally, another experiment has shown clear formation of regular filamentary plasma arrays propagating toward the microwave source [3].

Filamentary structures must be formed due to interactions between electromagnetic waves and charged particles and elastic and inelastic collision processes among the gas particles including neutrals. In order to find out an impact of filamentary structures on the thrust, it is necessary to understand the details of the structure of the plasma. However, it is difficult to analyze the ionization process experimentally. Thus we need numerical simulation with properly modeled atmospheric gas discharge. In this study, we have conducted PIC-MCC simulations under the atmospheric environment with an intense microwave and examined the formation of the filamentary structures.

\footnotetext{
${ }^{a}$ e-mail: ohnishi@rhd.mech.tohoku.ac.jp
}

This is an Open Access article distributed under the terms of the Creative Commons Attribution License 2.0, which permits unrestricted use, distribution, and reproduction in any medium, provided the original work is properly cited. 


\section{EPJ Web of Conferences}

\section{NUMERICAL METHODS AND SIMULATION CONDITIONS}

Formation of filaments may proceed in thermal and chemical non-equilibrium. In this study, therefore, we employ particle-in-cell (PIC) method to estimate interactions between charged particles and electromagnetic field, and Monte-Carlo collision (MCC) method is coupled with it for elastic and inelastic collisions between electrons and neutrals. In the PIC method, position and velocity of the particles are obtained by solving the equation of motion, and electromagnetic field is defined on a computational grid and calculated by solving Maxwell equations [4]. In cgs-Gauss system, the equation of motion of the charged particles in the electromagnetic field is described as follows;

$$
m \frac{d \mathbf{v}}{d t}=q\left(\boldsymbol{E}+\frac{\boldsymbol{v}}{c} \times \boldsymbol{B}\right),
$$

where $m, \mathbf{v}, q, c, \mathbf{E}$, and $\mathbf{B}$ are the mass of particle, the velocity of particle, the charge of particle, the speed of light, the electric field, and the magnetic field, respectively. Maxwell equations to be solved in our code are as follows;

$$
\begin{gathered}
\frac{1}{c} \frac{\partial \boldsymbol{E}}{\partial t}=\nabla \times \boldsymbol{B}-\frac{4 \pi}{c} \boldsymbol{J}, \\
\frac{1}{c} \frac{\partial \boldsymbol{B}}{\partial t}=-\nabla \times \boldsymbol{E},
\end{gathered}
$$

where $\mathbf{J}$ is the current density. In the PIC method, each computed particle is treated as a particle which acts as many real particles and has a fictitious large mass and charge. We give a finite size to the superparticle and distribute their charge and current density to each grid point with a weighting method. Since numerous super-particles are needed to reduce numerical errors, the equation of motion is parallelly computed by partitioning the particles to optimize CPUs. Additionally, for solving Maxwell equations, we have installed a parallel version of incomplete Cholesky conjugate gradients method.

In the MCC method, a collision probability is calculated with sample particles at each time step to reproduce elastic and inelastic collisions between electrons and neutrals. The collision probability of $k$-th particle is determined by $P_{k}=N \sigma_{l}(\epsilon) g_{k} \Delta t$, where $N$ is the neutral number density, $\sigma_{l}(\epsilon)$ is the cross section for collisional energy $\epsilon$ in $l$-th type of collision, and $g_{k}$ is the relative velocity between $k$-th particle and a neutral. If the collision probability is higher than a random number, the corresponding collision occurs, and the change in velocity of $k$-th particle is calculated in a stochastic manner. In the present paper, we consider only the two following reactions in the MCC step for simplicity:

$$
\begin{aligned}
& \mathrm{e}+\mathrm{N}_{2} \longleftrightarrow \mathrm{e}+\mathrm{N}_{2}, \\
& \mathrm{e}+\mathrm{N}_{2} \longrightarrow 2 \mathrm{e}+\mathrm{N}_{2}^{+},
\end{aligned}
$$

where Eq. (4) is the elastic collision, and Eq. (5) is the electron impact ionization.

In order to examine propagation of an ionization front, we set an initial breakdown spot for finding a process of creating a new spot by an intense microwave because we assume that the first breakdown occurs at a focal point in a microwave thruster, and subsequent discharged spots are created by the incident microwave reflected from it. Computational domain is a rectangle of $5 \mathrm{~mm} \times 5 \mathrm{~mm}$, and number of grid points is $1000 \times 1000$ for pure nitrogen gas. A hot circle spot is set in the center region as an initial breakdown. Its diameter is $1 \mathrm{~mm}$. Charged particles in the spot consist of electron and $\mathrm{N}_{2}^{+}$, and their initial number density is $5.0 \times 10^{14} \mathrm{~cm}^{-3}$. Initial velocity of the charged particles in the spot is randomly set as a Maxwellian distribution at $10,000 \mathrm{~K}$. The corresponding plasma frequency of the spot is $2.0 \times 10^{11} \mathrm{~s}^{-1}$, and the skin depth is $0.2 \mathrm{~mm}$. Neutral species $\mathrm{N}_{2}$ is not treated as particles but as a continuous medium at atmospheric pressure, ambient temperature $(300 \mathrm{~K})$, with number density of $2.0 \times 10^{19} \mathrm{~cm}^{-3}$. From the left boundary $(x=0 \mathrm{~mm})$, an incident microwave travels to the right in Fig. 1. The intensity is $5.0 \mathrm{MV} / \mathrm{m}$, and the frequency is $110 \mathrm{GHz}$. Electric and magnetic fields 

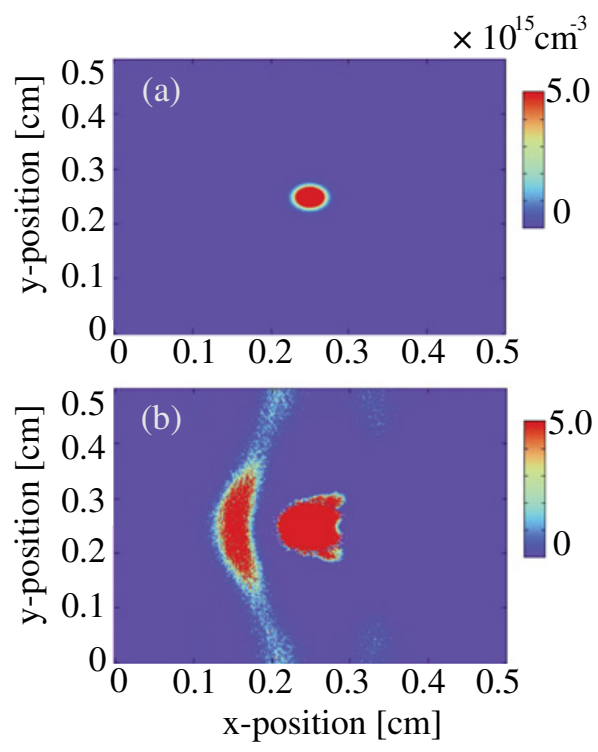

Figure 1. Contours of electron number density at (a) $t=0$ and (b) $t=1.3 \mathrm{~ns}$.

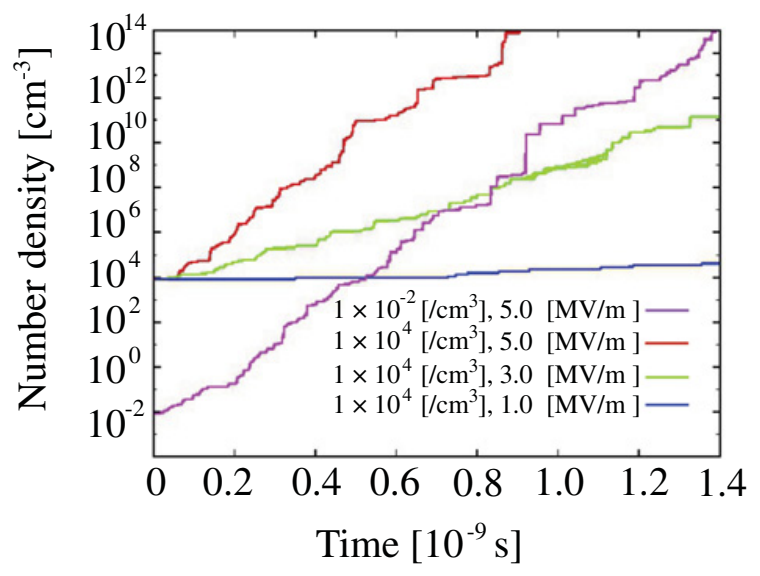

Figure 2. Time evolution of electron number density at the newly created ionization front with different conditions.

are damped smoothly in $5 \%$ from the right boundary $(x=4.75-5 \mathrm{~mm})$ to avoid a reflection of the microwave from the boundary.

\section{RESULTS AND DISCUSSIONS}

Once the incident microwave reaches the hot spot, it is reflected and scattered at the surface of the spot. Then the amplitude of the electric field raises up above the original amplitude ahead of the surface. The region of strong field is discretisely formed at a quarter of microwave wavelength from the original spot. Figure 1 shows contours of the electron number density. The initially given plasma spot spreads while absorbing the microwave energy. Additionally, a newly ignited region is created ahead of the original spot through electron impact ionization originating in the background electrons. Since the intensity of the electric field is amplified due to the existence of the original spot, the distance between the new spot and the original one is a quarter of the microwave wavelength and is consistent with the past studies [5]. The electron number density of the new spot exponentially grows to the level of the original one in $1.3 \mathrm{~ns}$ (Fig. 1(b)), and we can consider that the ionization front moves to the left side in this duration. This indicates that the propagating speed of the ionization front is about $400 \mathrm{~km} / \mathrm{s}$ which is one order of magnitude larger than the measured one. The major reasons for this discrepancy may be higher number density of the background electrons and higher intensity of the incident microwave as discussed below.

Figure 2 shows time evolution of number density at the new ionization front for the case of different initial number densities of the background electron. Red and purple lines are for the cases of the initial number densities of $1.0 \times 10^{4} / \mathrm{cm}^{3}$ and $1.0 \times 10^{-2} / \mathrm{cm}^{3}$, respectively, with $5.0 \mathrm{MV} / \mathrm{m}$ EM field, and blue and green lines are for the cases of $1.0 \times 10^{4} / \mathrm{cm}^{3}$ with $1.0 \mathrm{MV} / \mathrm{m}$ and $3.0 \mathrm{MV} / \mathrm{m}$, respectively. We can find that the electron number density grows exponentially for all cases, and the growth rates are the same for all situations with identical microwave intensity. Even if the number density of the background electrons is sufficiently low at atmospheric environment and room temperature, the new ionization front is formed on a ns timescale while the experimental studies suggested the front should move to the new 


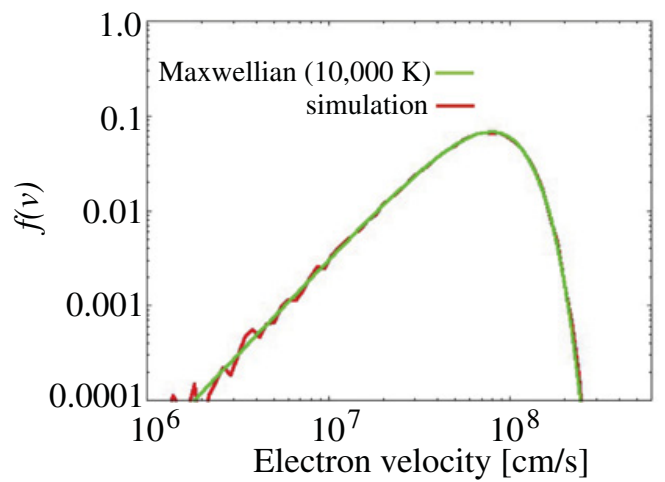

(a) initial condition

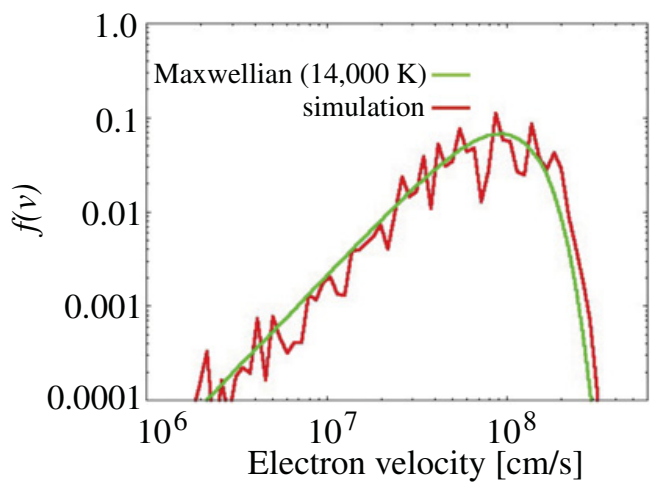

(b) $1.3 \mathrm{~ns}$

Figure 3. Velocity distribution $f(v)$ at (a) the initial and (b) the next ionization front.

one in a ten ns timescale [3]. On the other hand, the influence of the incident microwave intensity is relatively strong on forming the ionization front. For the case of $1.0 \mathrm{MV} / \mathrm{m}$ EM field, the growth rate is much smaller than that of $5.0 \mathrm{MV} / \mathrm{m}$. It takes a couple of ten ns for creating a new front, and the front propagation speed might be of the same order as the experimental one.

Actually, the fitting formula for electron density growth can be obtained as a function of the field intensity from the simulation results;

$$
n_{e}=5.5 \exp \left(7.2 \times 10^{8} E^{2} t\right)
$$

where $n_{e}$ and $E$ are the electron density in $\mathrm{cm}^{-3}$ and the electric field amplitude in $\mathrm{MV} / \mathrm{m}$, respectively. The formula suggests that the speed of ionization front propagation is proportional to $E^{2}$ because it must be determined by the timescale of the formation of the ionization front. This tendency agrees well with the experimental results. At atmospheric pressure, the collision frequency is $3.5 \times 10^{12} \mathrm{~s}^{-1}$ and is much higher than that of the incident microwave. So, the electron energy increases with time in proportion to $E^{2}[6]$.

In a past simulation study, Chaudhury et al. pointed out that the propagation of the ionization front is determined not only by the electron impact ionization but also by the electron diffusion through their plasma fluid simulations [7]. However, in our particle simulations, we have hardly found any clear indication that the electron diffusion plays an important role in the front propagation. One possible reason for this difference is the initial setting of the spot temperature. Although they assumed that the initial plasma spot is created at $2 \mathrm{eV}$, we set it to a slightly lower value of $10,000 \mathrm{~K}$. Both settings are not validated at present while the newly created spot develops in the temperature of $14,000 \mathrm{~K}$ in our simulation (Fig. 3(b)). The spot temperature must result from large cross-section of e- $\mathrm{N}_{2}$ collision in the collision energy range of $1-3 \mathrm{eV}$. Further simulations may resolve this issue since they can determine the required temperature to maintain the front propagation.

\section{CONCLUSIONS}

We have developed a numerical code to reproduce an ionization front with intense microwave propagation using the PIC method coupled with the MCC method for collisional processes among charged particles and neutrals. The results show that the incident microwave reflects at the surface of the initial discharge spot, and the field strength near the spot reaches a value higher than the initial one. Thus the newly ignited spot grows at this region through the electron impact ionization originating in the background electrons. However, the resulting creation speed of the new discharge spot is higher than the measured one. A careful investigation of contributions arising from incident microwave and 


\section{IFSA 2011}

temperature of the initial hot spot should be performed experimentally and numerically to assert more reliable predictions.

\section{References}

[1] Y. Oda et al., J. Appl. Phys. 100, 113307 (2006)

[2] Y. Oda et al., J. Plasma Fusion Res. 83, 296 (2007)

[3] Y. Hidaka et al., Phys. Plasmas 16, 055702 (2009)

[4] C. K. Birdsall and A. B. Langdon, Plasma Physics via Computer Simulation (Taylor and Francis, New York, 2004)

[5] Y. Hidaka et al., Phys. Rev. Lett. 100, 035003 (2008)

[6] Y. P. Raizer, Gas Discharge Physics (Springer-Verlag, Berlin, 1991)

[7] B. Chaudhury et al., Phys. Plasmas 17, 123505 (2010) 